\title{
Structure and evolution of W UMa-type systems
}

\author{
Lifang $\mathbf{L i}^{1}$, Fenghui Zhang ${ }^{1}$, Zhanwen Han $^{1}$, Dengkai Jiang ${ }^{1,2}$ \\ and Tianyu Jiang ${ }^{1,2}$ \\ ${ }^{1}$ National Astronomical Observatories Yunnan Observatory, Chinese Academy of Sciences, \\ P. O. Box 110, Kunming, Yunnan Province, 650011, P.R. China \\ email: gssephd@public.km.yn.cn .or. llf@ynao.ac.cn \\ ${ }^{2}$ Graduate University of Chinese Academy Sciences, Beijing 100039, China
}

\begin{abstract}
We summarize and discuss our recent works on the structure and evolution of lowmass W UMa-type contact binary stars. Three conclusions are given as followings: (1) The energy transfer is taken place in the radiative region of common envelope of W UMa systems; (2) The magnetic activity level of W UMa systems is weaker than that of non-contact binaries or rapid-rotating single stars; (3) The evolutionary outcome of W UMa systems might be the rapid-rotating single stars, and an average lifetime is derived to be about 7 Gyr for W UMa systems.
\end{abstract}

Keywords. binaries: close, stars: evolution, stars: rotation, stars: activity.

\section{Introduction}

W UMa binaries are very common eclipsing binaries in solar neighbourhood and are classified into A- and W-type systems. A-subtype W UMa stars show primary minima resulting from the eclipse of the larger, more massive component, whereas the opposite is true for $\mathrm{W}$-subtype ones. The pioneer works on the structure and evolution of contact binaries were carried out by Lucy (1976) and Robertson \& Eggleton (1977). This issue has been recently investigated by many authors (Li, Han \& Zhang 2004,2005; Yakut \& Eggleton 2005, etc). Serious uncertainties on evolution of contact binaries concern the energy transfer (Kahler 2002) and the evolutionary outcome. In this paper, we summarize our recent works on the structure and evolution of W UMa contact binaries.

\section{The models}

It is not yet clear where and how the energy is transferred in W UMa systems. Li, Han \& Zhang (2004) constructed a conservative model to investigate the energy transfer in W UMa systems using Eggleton's code (Eggleton 1971). The results are shown in Figure 1. It is seen in Fig. 1 that the model exhibits the observational properties of A-subtype systems if energy transfer occurs in the base or the whole of common envelope, and that the model shows the observational properties of W-subtype systems if the energy transfer occurs in the outermost layers. This suggests that the energy transfer in W UMa systems might be taken place in the radiative region of common envelope.

Li, Han \& Zhang (2005) investigated non-conservative evolution of low-mass W UMa contact binaries with angular momentum (AM) loss owing to magnetic stellar wind (MSW) (Hurley et al 2002). The time dependence of some quantities are shown in Figure 2. As seen from figure 2 that W UMa contact binaries would merge into a fast-rotating single star owing to Darwin's instability (Eggleton \& Kiseleva-Eggleton 2001) after about 

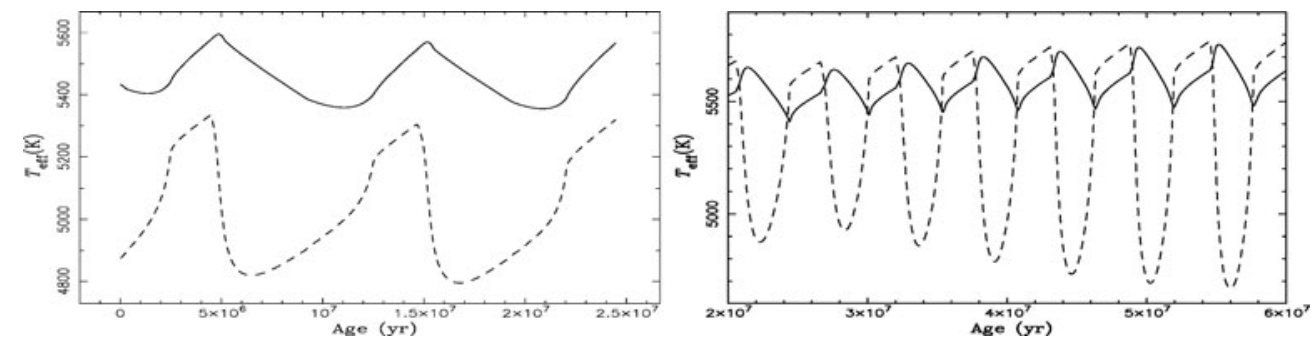

Figure 1. The time dependence of the effective temperature of the primary (solid line) and the secondary (dashed line). Left panel shows the models in which the energy transfer is taken placed in the base or in the whole of the common envelope, and right panel shows the model in which the energy transfer is taken placed in the outmost layers of the common envelope.
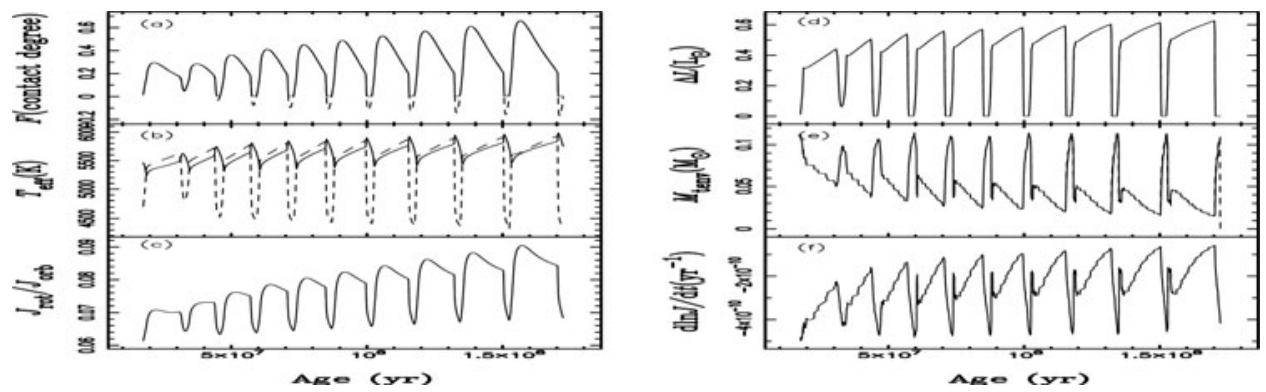

Figure 2. The time dependence of contact degree, $F$, the effective temperature of the primary (solid line) and the secondary (dashed line), together with the ratio of the rotating angular momentum to the orbital angular momentum, the luminosity transfer rate, the total mass contained in convective envelopes $\left(M_{\text {tenv }}\right)$, and the rate of angular momentum loss ( $\mathrm{Li}$, Han \& Zhang 2005).

1200 cycles (about 7Gyr), and that the rate of AM loss in contact evolution is much lower than that in non-contact stage, which is very consistent with the result predicted by observations (Stepien et al. 2001, and references therein) and a polytropic model of Rucinski (1992). This might be caused by less total mass contained in the convective envelopes of contact binaries, comparing with that of non-contact binaries.

\section{Acknowledgements}

This work was partly supported by the Chinese Natural Science Foundation (10673029, 10773026 and 10433030), and by the Yunnan Natural Science Foundation (2007A113M and 2005A0035Q).

\section{References}

Eggleton, P. P., 1971, MNRAS, 151, 351

Eggleton, P. P. \& Kiseleva-Eggleton, L., 2001, ApJ, 562, 1012

Hurley, J. R., Tout, C. A., \& Pols, O. R., 2002, MNRAS, 329, 897

Kähler, H., 2002, A\&ٌA, 395,899

Li, L., Han, Z., \& Zhang, F., 2005, MNRAS, 351, 137

Li, L., Han, Z. \& Zhang, F., 2005, MNRAS, 360, 272

Lucy, B., 1976, ApJ, 205, 208

Robertson, J. A. \& Eggleton, P. P., 1977, MNRAS, 179, 359

Rucinski, S. M., 1992, AJ, 103, 960

Stȩpień, K., Schmitt, H. M. M., \& Voges, W., 2001, A\& A, 370, 157

Yakut, K. \& Eggleton, P. P., 2005, ApJ , 629, 1055 